\title{
Numerical Simulation on flow characteristics of the supercritical fluid in Micro-Fin Tube
}

\author{
Chen Peng-Fei ${ }^{1}$, Chen Kang ${ }^{1,2, *}$, Ma Gao-xiang ${ }^{1}$, Wang Xiao ${ }^{1}$, and Wen Long ${ }^{1}$ \\ ${ }^{1}$ Institute of solar engineering technology, Northwest Engineering Corporation Limited, Power China, 710065, Xi'an China; \\ ${ }^{2}$ Power China Research Center of Solar Thermal Power Engineering Technology, 710065, Xi'an China
}

\begin{abstract}
The flow characteristics of the supercritical fluid in the micro-fin tube is the theoretical basis for the development of heat transfer enhancement and flow resistance reduction in the micro-fin tube. For micro-fin tubes with different fin shapes, this paper considered the physical properties of nitrogen in the supercritical state and adopted the enhanced wall function, reasonable turbulence equations, and control equations, etc., to numerically simulate the flow of nitrogen in a $2 \mathrm{~mm}$ micro-fin tube under supercritical pressure. The distribution of velocity field, turbulence and pressure field of supercritical nitrogen in the micro-fin tube was analyzed. The turbulent flow mechanism of the micro-fins was obtained, and it is found that the existence of the viscous bottom layer slows down the inter-costal fluid velocity and increases the frictional resistance during the flow process.
\end{abstract}

\section{Introduction}

With the rapid development of solar thermal power generation technology, aviation technology, microelectronic technology, and semiconductor industry, heat exchangers have begun to develop towards miniaturization, and more and more millimeter-level heat exchange components have appeared, the traditional relational formula of cooling technology and heat transfer can no longer satisfy the needs of new heat exchange equipment.

The miniaturization of the heat exchange tube size can effectively improve the heat transfer performance of the heat exchanger, but the processing technology of the heat exchange tube limits the further reduction of the tube diameter. Therefore, small-scale spoilers such as inner fin tubes like micro-fin tubes and fluted tubes, and in-tube inserts including metal foam and twisted bands can achieve the design of the heat exchanger with high efficiency, compactness and light weight ${ }^{[1-4]}$.

Many scholars have conducted a lot of research on the influence of the geometric parameters of the microfin tube on the heat transfer effect. Kimura ${ }^{[5]}$ studied the effect of the helix angle of the micro-fin tube; in 1985, Shinohaya, Toke ${ }^{[6]}$ and other researchers proposed the optimal helix angle of the micro-fin tube with different geometric structures. Jensen ${ }^{[7]}$ believed that with the increase of $N, H$, and $\alpha$, the frictional resistance gradually increases. But Jensen noticed that when $H=0.02$, the resistance was larger than when $H=0.03$, which was inconsistent with expectations. It is considered that the flow velocity in the smaller area between the fins is affected by the viscous substrate, so it has a smaller velocity than that in the high-fin tube. This smaller speed will cause the friction factor to decrease. Increasing the fin height or reducing the number of fins, the friction coefficient does not increase much. The influence of $N, H$, and $\alpha$ on $N u$ is consistent with the influence on friction factor. The helix angle has a greater impact on high-fin tubes than low-fin tubes. For high-fin tubes, when the helix angle changes from $0^{\circ} \sim 30^{\circ}$, the $N u$ number increases by about $30 \sim 60 \%$; for the micro-fin tube, when the helix angle changes from $15^{\circ} \sim 45^{\circ}, \mathrm{Nu}$ only increases by $30 \sim 40 \%$. He believes that Carnavos 's correlation formula ${ }^{[8]}$ has a large error, especially when $\alpha>30^{\circ}$. The friction factor of the high-fin tube is $40 \sim 170 \%$ of that of the smooth tube, while the friction factor of the micro-fin tube is $40 \sim 140 \%$. The $N u$ of highfin tubes increased by $50 \sim 150 \%$, while the micro-fin tubes increased by $20 \sim 220 \%$.

Sui ${ }^{[9]}$ and others of Tsinghua University performed a numerical simulation of the turbulent flow in an inner micro-fin tube, and at the same time performed a numerical study on the performance of rectangular, triangular, and semicircular fins under the same conditions of other parameters. He believes that the increase in the number of fins can generate additional spiral flow, but it should not be too high. A narrow fin spacing will cause fluid retention, thereby inhibiting heat transfer. At the same time, the heat transfer of rectangular fins is better than that of spiral and triangular micro-fin tubes.

In summary, the current flow characteristics in micro-fin tubes are limited to incompressible fluids, and there are few studies on supercritical fluids. In this paper, the numerical simulation of supercritical fluid in microfin tube provides the basis for the extended application

Corresponding author: chen_kang@xjtu.edu.cn 
of micro-fin tube in the field of heat transfer enhancement.

\section{Numerical model and methods}

\subsection{Physical model}

To study the turbulent flow and heat transfer of supercritical nitrogen in horizontal micro-fin tubes, a heat exchange tube with an outer diameter of $2 \mathrm{~mm}$, a wall thickness of $0.05 \mathrm{~mm}$, and a tube length of $100 \mathrm{~mm}$ was selected for numerical simulation (as shown in Figure 1), where the cold fluid flows from the inside to the outside along the positive direction of the $Z$ axis. Since the outer diameter of the pipeline is only $2 \mathrm{~mm}$, the hydraulic diameter of the flow section is less than $1.90 \mathrm{~mm}$, and the inlet velocity is high, the influence of buoyancy can be ignored in this paper.

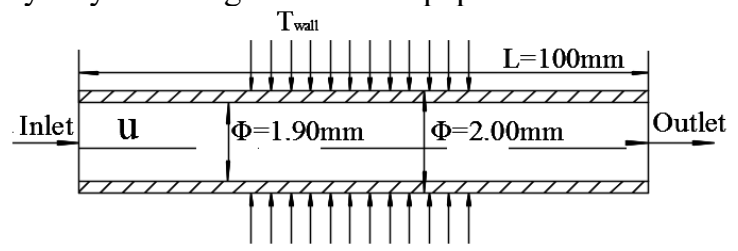

(a) $\mathrm{Z}$ axis

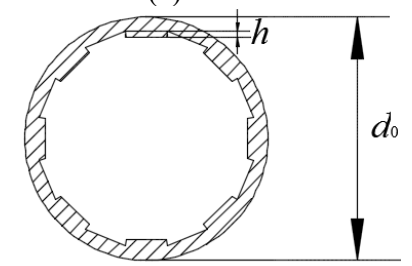

(b) XY axis

Fig. 1. Geometric model of micro-rib tube.

The physical model parameters of the micro-fin tube and the smooth tube used in this paper are shown in the following table.

Table 1. Dimensions of different micro-rib tubes.

\begin{tabular}{ccccc}
\hline $\begin{array}{c}\text { Geometrical } \\
\text { dimension }\end{array}$ & $\begin{array}{c}\text { Inner } \\
\text { diameter } \\
d_{i} / \mathrm{mm}\end{array}$ & $\begin{array}{c}\text { Outer } \\
\text { diameter } \\
d_{0} / \mathrm{mm}\end{array}$ & $\begin{array}{c}\text { Rib } \\
\text { height } \\
h / \mathrm{mm}\end{array}$ & $\begin{array}{c}\text { Rib } \\
\text { number } \\
N\end{array}$ \\
\hline $\begin{array}{c}\text { Smooth tube } \\
\text { Square straight } \\
\text { micro-rib tube }\end{array}$ & 1.8 & 2 & 0 & 0 \\
$\begin{array}{c}\text { Square straight } \\
\text { micro-rib tube }\end{array}$ & 1.8 & 2 & 0.05 & 8 \\
$\begin{array}{c}\text { Square straight } \\
\text { micro-rib tube }\end{array}$ & 1.8 & 2 & 0.05 & 16 \\
$\begin{array}{c}\text { Square straight } \\
\text { micro-rib tube }\end{array}$ & 1.8 & 2 & 0.05 & 32 \\
$\begin{array}{c}\text { Square straight } \\
\text { micro-rib tube }\end{array}$ & 1.8 & 2 & 0.1 & 16 \\
$\begin{array}{c}\text { Square straight } \\
\text { micro-rib tube } \\
\text { Triangular }\end{array}$ & 1.8 & 2 & 0.4 & 16 \\
$\begin{array}{c}\text { straight micro- } \\
\text { rib tube }\end{array}$ & 1.8 & 2 & 0.05 & 16 \\
\hline
\end{tabular}

\subsection{Governing equation}

The governing equation is composed of threedimensional steady-state incompressible continuity equation, Reynolds average Navier-Stokes equation and energy equation.

Continuity equation:

$$
\nabla \cdot(\rho u)=0
$$

Among them, $\rho$ is the density of the fluid, and $u$ is the velocity of the fluid.

Momentum conservation equation:

$$
\frac{\partial}{\partial x_{j}}\left(\rho u_{i} u_{j}\right)=-\frac{\partial p}{\partial x_{i}}+\frac{\partial}{\partial x_{i}}\left[\left(\mu+\mu_{t}\right)\right]
$$

\subsection{Boundary conditions}

The nitrogen inlet velocity in the tube is $4 \mathrm{~m} \cdot \mathrm{s}^{-1}$, the inlet pressure is $100 \mathrm{bar}$, the inlet temperature is $188 \mathrm{~K}$, the constant wall temperature boundary condition is set to $300 \mathrm{~K}$, the outlet is the pressure outlet, and there is no slip boundary condition.

Taking into account the actual heat transfer situation, the boundary conditions of this paper make the following assumptions:

(1) The flow of fluid in the tube belongs to single-phase heat transfer without phase change;

(2) The inside of the tube is real gas, and the NIST physical property model is used to measure the physical property parameters of N2;

(3) The effect of gravity is not considered in this paper;

(4) The fluid is in a convective heat transfer condition with fully developed turbulent flow.

The equivalent diameter in the text is defined as:

$$
D_{h}=\frac{4 A}{P}
$$

Where $\mathrm{A}$ is the cross-sectional area of circulation $\left(\mathrm{m}^{2}\right)$; $\mathrm{P}$ is the wet circumference $(\mathrm{m})$.

Where $\Delta p$ is the average pressure drop in the calculation area of the micro-fin tube $(\mathrm{Pa})$.

\section{Experimental results and discussion}

This paper mainly studied the influence of micro-fin tube structure on supercritical nitrogen turbulent heat transfer, and analyzed the influence of different fin shapes including triangular straight micro-fin tubes and square straight micro-fin tubes. The internal relationship between the fin shape parameters and the heat transfer characteristics of supercritical nitrogen flow was obtained. 


\subsection{Turbulence characteristics analysis}

Figure 2 shows the distribution of turbulence along the axis under different fin shapes. It can be seen that the turbulence of the triangular straight micro-fin tube is obviously higher than that of the smooth round tube and the square straight micro-fin tube under the same conditions. The turbulence degree of the three tube types is very different. The turbulence degree of the square straight micro-fin tube changes smoothly when $\mathrm{z}$ is larger than $50 \mathrm{~mm}$, indicating that the flow has entered the region where turbulence is fully developed. At the same time, the shape of the triangular fin tube has a very obvious disturbance to the flow. From this point of view, in the area close to the wall, the heat transfer of the triangular micro-fin tube is better than that of the square straight micro-fin tube and the smooth round tube.

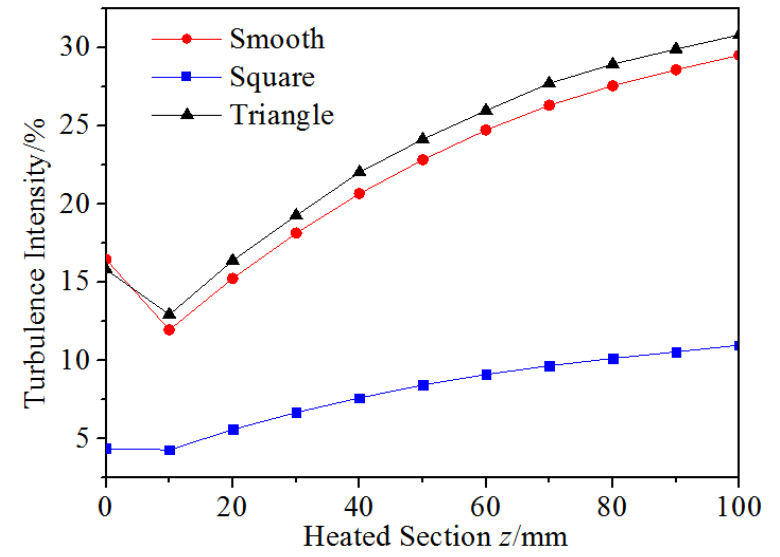

Fig. 2. The distribution of turbulence along the axis under different fin shapes

Figure 3 shows the turbulence distribution cloud diagram of different fin shapes at $z=60 \mathrm{~mm}$. It can be seen from the figure that the turbulence distribution of the square straight micro-fin tube on the $\mathrm{z}=60 \mathrm{~mm}$ section is relatively uniform with a smaller gradient, followed by the smooth tube, and the turbulence uniformity distribution in the triangular straight microfin tube is the worst. In the vicinity of the wall, the turbulence of the square straight micro-fin tube is relatively large, and the turbulence of the inter-fin and fin-tip does not change much; the turbulence of the triangular straight micro-fin tube at the fin valley is not uniformly distributed in the circumferential direction, the turbulence on both sides of the fin valley is relatively large, and the turbulence in the middle area of the fin valley is relatively small; the smooth circular pipe has high turbulence distribution only at $45^{\circ}, 135^{\circ}, 225^{\circ}$ and $315^{\circ}$. These results show that the existence of straight micro-fins strengthens the turbulence at the wall surface and produces flow separation, especially at the roots of the fins. The turbulence gradient of square micro-fin tubes is smaller than that of triangular and smooth tubes. At the tip of the rib, the turbulence gradient of the square micro-fin is larger than that of the triangular and smooth tube. Therefore, the fluid in the wall area can better contact the fluid in the turbulent core area, and the convective heat transfer capability is enhanced.

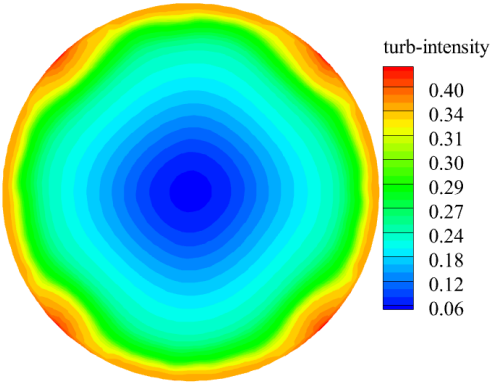

(a) Triangular fins

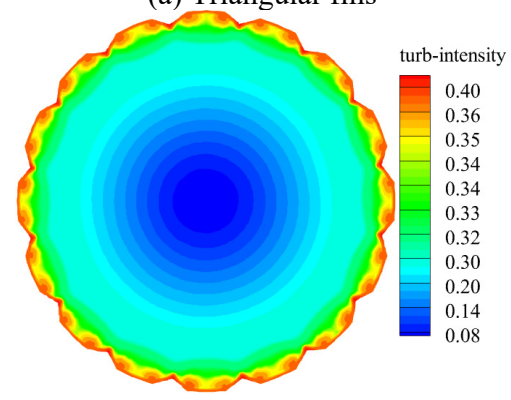

(b) Square fins

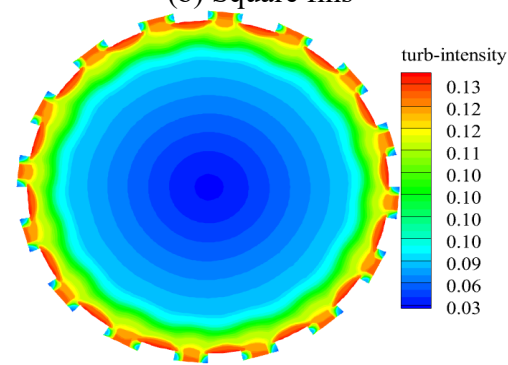

(c) Smooth tube

Fig. 3. Turbulence distribution cloud diagram of finned tubes with different shapes at $\mathrm{z}=60 \mathrm{~mm}$

\subsection{Analysis Pressure field analysis}

Figure 4 shows the average pressure distribution under different shapes. It can be seen that the pressure drop of the square straight micro-fin tube is very obvious, the inlet and outlet pressure difference is $4796 \mathrm{~Pa}$, while the pressure of the smooth tube and the triangular tube have dropped by $2676 \mathrm{~Pa}$ and $2806 \mathrm{~Pa}$, respectively. Compared with the smooth tube, the pressure difference of the triangular micro-fin tube only increased by $4.76 \%$, while the square tube did increase by $79.22 \%$, and the pressure loss was huge. From the calculation results, the maximum pressure drop is $4796 \mathrm{~Pa}$, and the fluid is still in a supercritical state.

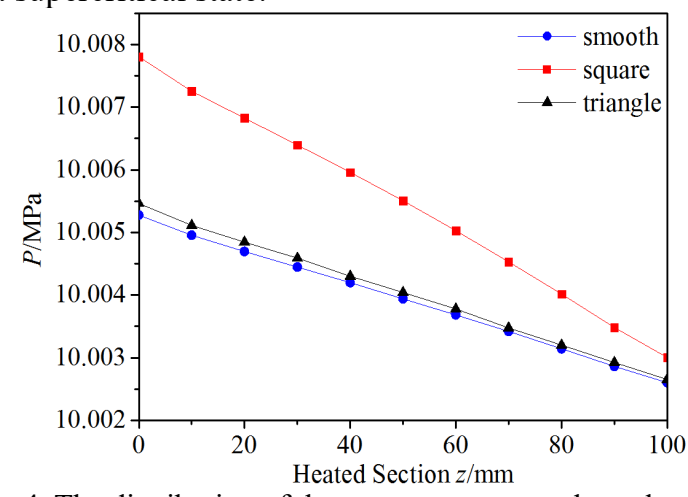

Fig. 4. The distribution of the average pressure along the axial direction under different fin shapes 
Figure 5 shows the distribution of $\mathrm{x}=0 \mathrm{~mm}$ pressure along the axis under different fin shapes. At the inlet, the pressure drops extremely along the axis, and the pressure gradient in the radial direction is larger; at the outlet, the pressure distribution of the square straight micro-fin tube is more uniform than that of the triangular straight micro-fin tube and the smooth round tube.

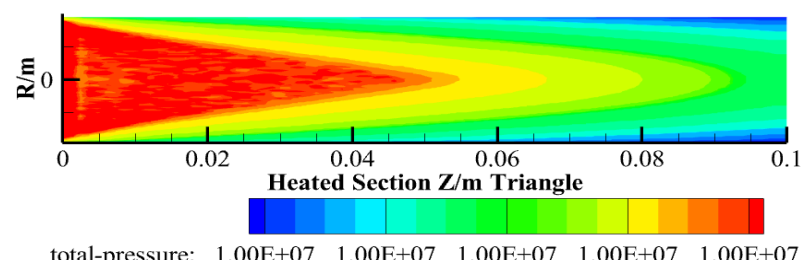

(a) Triangular fins

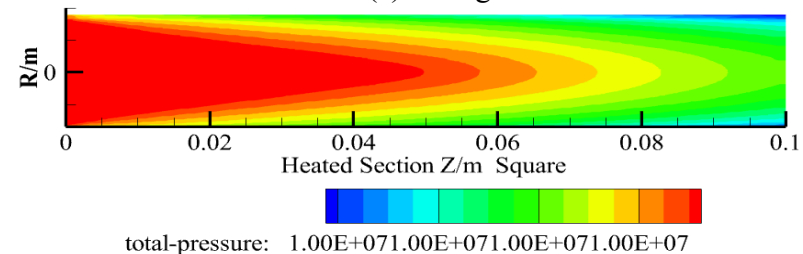

(b) Square fins

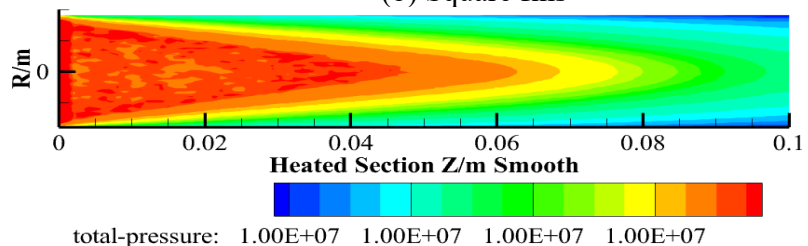

(c) Smooth tube

Fig. 5. The distribution of pressure along the axis under different fin shapes at $\mathrm{x}=0 \mathrm{~mm}$

Regarding the pressure distribution on the axis, it can be seen that although the introduction of fins can enhance the heat transfer capacity, the increase in heat transfer capacity also brings some negative factors, that is, the continuous increase in pressure loss and the increase in friction resistance.

\subsection{Analysis analysis of the velocity field in the tube}

To analyze the distribution of the fin shape on the fluid velocity field in the turbulent heat transfer process, this paper intercepted the velocity cloud diagram at $\mathrm{z}=60 \mathrm{~mm}$, and revealed the influence of the inter-fin and fin-tip velocity changes on the turbulent heat transfer.

Figure 6 shows the velocity distribution cloud diagram of the supercritical fluid under different tube types at the $\mathrm{x}=0 \mathrm{~mm}$ section. It can be seen from the figure that the velocity distribution of the fluid in the different tube types at the $x=0 \mathrm{~mm}$ section is similar. The velocity distribution of the triangular straight micro-fin tube and the square straight micro-fin tube is the same. The difference is that the speed of the square straight micro-fin tube in the turbulent core area is higher than that of the triangular straight micro-fin tube and the smooth round tube. The speed gradient of the ribbed tube and the square straight micro-fin tube at the axis is larger than that of the smooth round tube, which increases the heat transfer.

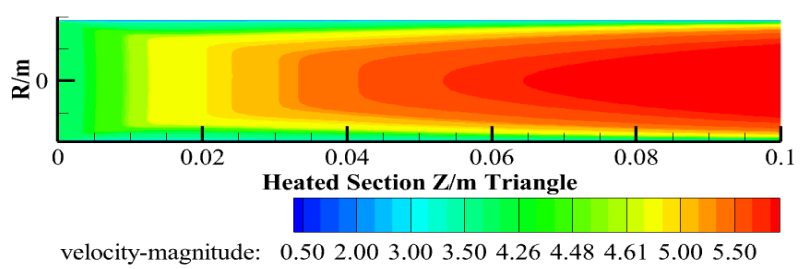

(a) Triangular fins

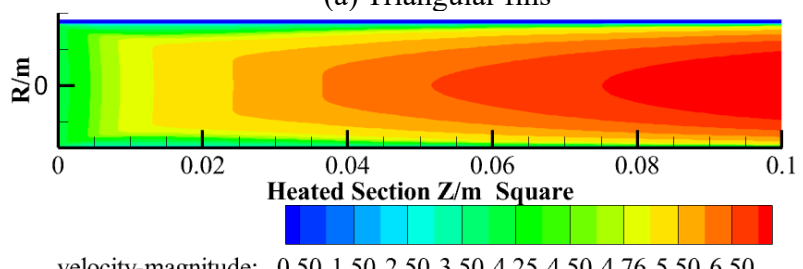

(b) Square fins

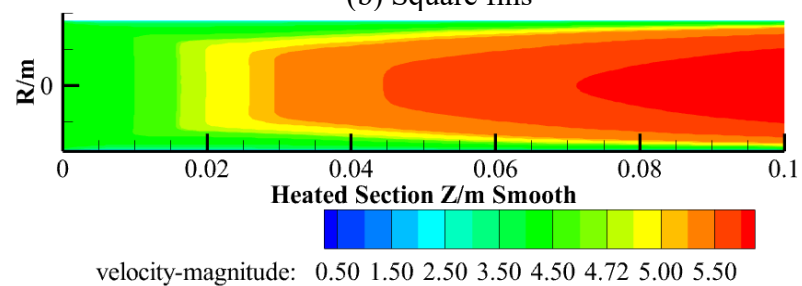

(c) Smooth tube

Fig. 6. The velocity distribution along the axis at the $x=0 \mathrm{~mm}$ section under different fin shapes

The presence of straight micro-fins changes the velocity field distribution of supercritical nitrogen flowing in the tube. The increase in the radial velocity gradient enables the low-temperature fluid at the central axis to quickly exchange heat with the high-temperature fluid near the wall, enhancing the turbulent heat transfer ability of the fluid, which has a one-to-one correspondence with the temperature gradient change at $\mathrm{z}=60 \mathrm{~mm}$ at the axis analyzed above; in the micro-fin tube, due to the presence of fins, the velocity boundary layer at the wall is destroyed, the velocity of the fluid at the same radial height is greater than that of a smooth tube, that is, the velocity gradient of the square straight micro-fin tube is greater than that of the triangular straight micro-fin tube; the intercostal fluid is affected by the viscous bottom layer, making the velocity much smaller. In the area near the wall, the thickness of the velocity boundary layer at the tip of the fin is significantly smaller than that at the top of the rib, which results in a greater change in velocity at the tip of the fin and a thinner boundary layer, and enhances the heat exchange between the fluid and the wall.

\section{Conclusion}

This paper discussed the influence of different fin shapes on the flow process of cryogenic nitrogen in a supercritical pressure tube, and conducted a detailed numerical simulation study to analyze the velocity field, turbulence and pressure field distribution of supercritical nitrogen in micro-fin tube.

The introduction of micro-fins in the tube can change the velocity field of the supercritical fluid, which makes the fluid flow in the tube oscillate, and the turbulence $I$ increases, resulting in the enhancement of heat transfer capacity; the introduced fins reduce the flow velocity of 
intercostal fluid and thicken the boundary layer; when the fluid flows in the micro-fin tube, due to the existence of the viscous bottom layer, the speed of the inter-costal fluid slows down, which increases the frictional resistance during the flow process and causes a sharp drop in pressure.

\section{Acknowledgement}

This work was supported by Natural Science Basic Research Program of Shaanxi (Grant No.2020JQ-1001).

\section{References}

1. Chen K, Guo L, Xie X, et al. Experimental investigation on enhanced thermal performance of staggered tube bundles wrapped with metallic foam $[\mathrm{J}]$. International Journal of Heat \& Mass Transfer, 2018, 122: 459-468.

2. Chen K, Guo L, Wang H. A review on thermal application of metal foam [J]. Science China Technological Sciences, 2020, https://doi.org/10.1007/s11431-020-1637-3

3. Chen Kang, Chen Pengfei, Li Wensheng, et al. Permeation flow characteristics in electrodeposited metal foams[J]. Journal of Xi'an Jiaotong University, 2020, 54(5): 87-94

4. Chen Kang, Guo Liejin, Zhao Liang, et al. Experimental study on flow and heat transfer characteristics of metal foam tube bundles[J]. Journal of Engineering Thermophysics, 2016, 37(4): 770-774.

5. Ito M, Kimura H. Boiling Heat Transfer and Pressure Drop in Internal Spiral-Grooved Tubes[J]. Bulletin of Jsme, 1979, 22(171):1251-1257.

6. Shinohara Y, Tobe M. Development of an Improved Thermofin tube[J]. Hitachi Cable Review, 1985, 4: 47-50.

7. Kim J H, Jansen K E, Jensen M K. Simulation of three-dimensional incompressible turbulent flow inside tubes with helical fins[J]. Numerical Heat Transfer, Part B, 2004, 46(3): 195-221.

8. Carnavos $\mathrm{T}$ C. Heat Transfer Performance of Internally Finned Tubes in Turbulent Flow[J]. Heat Transfer Engineering, 2007, 1(4):32-37.

9. Sui Xiaofeng, Meng Ji'an, Chen Zejing, etc. Threedimensional numerical simulation of convective heat transfer in spirally finned tubes[J]. Journal of Engineering Thermophysics, 2005, 26(2): 292-294. 